\title{
The use of over-the-counter analgesics in patients with chronic kidney disease
}

\author{
Marzena Jakimowicz-Tylicka, Michał Chmielewski (D), Izabella \\ Kuźmiuk-Glembin (D), Piotr Skonieczny (D) , Grażyna Dijakiewicz, \\ Grażyna Zdrojewska, Bolesław Rutkowski, Leszek Tylicki \\ Alicja Dębska-ślizień
}

Department of Nephrology, Transplantology and Internal Diseases, Medical University of Gdańsk, Poland

\begin{abstract}
Background: Analgesics can be sold following medical prescription, but also as over-the-counter (OTC) medications. In patients with chronic kidney disease (CKD), their use could potentially be associated with increased risk of side-effects, due to impaired renal elimination. The aim was to evaluate the epidemiology and indications for the use of OTC analgesics, and the knowledge of their side-effects in patients with CKD.

Materials and methods: A cross-sectional, controlled survey on the use of OTC analgesic drugs was conducted among 180 CKD patients (stage 1-5, dialysis, kidney transplant), compared to 60 controls.

Results: The proportion of patients using OTC analgesics on a regular basis was higher in the CKD group, compared to controls ( $18.9 \%$ vs. $10.0 \%, \mathrm{p}<0.02)$. The major indications included musculoskeletal issues, followed by headaches and other. Subgroup analysis revealed that analgesic use was lowest among transplanted patients, in comparison to CKD stage $1-5$, and dialysis subjects $(10 \%, 20 \%, 26 \%$, respectively, $\mathrm{p}=0.06)$. Less than half of CKD patients and controls declared any knowledge on potential side-effects of analgesic drugs (45.6\% vs. $40.0 \%$, NS). Conclusions: The use of OTC analgesics among patients with CKD is higher than in subjects without CKD, with the exception of transplanted patients. The knowledge on the potential side-effect of analgesics is limited. Keywords: chronic kidney disease / analgesics / over-the-counter
\end{abstract}

\section{Citation}

Jakimowicz-Tylicka M, Chmielewski M, Kuźmiuk-Glembin I, Skonieczny P, Dijakiewicz G, Zdrojewska G, et al. The use of over-the-counter analgesics in patients with chronic kidney disease. Eur J Transl Clin Med. 2018;1(2):11-16.

DOI: 10.31373/ejtcm/99912

Corresponding author:

Michał Chmielewski, Department of Nephrology, Transplantology and Internal Diseases, Medical University of Gdańsk, Dębinki 7, 80-211, Gdańsk, Poland

e-mail: michal.chmielewski@gumed.edu.pl

No external funds.

Available online: www.ejtcm.gumed.edu.pl

Copyright ${ }^{\circledR}$ Medical University of Gdańsk

This is Open Access article distributed under the terms of the Creative Commons Attribution-ShareAlike 4.0 International. 


\section{Introduction}

Analgesics are drugs administered to achieve relief from pain. They are typically prescription-only but many of them are also sold as over-the-counter (OTC) medications, i.e. without a prescription from a healthcare professional. Analgesics are among the most popular and best-selling OTC drugs, as according to some studies they account for $40-50 \%$ of all the medications sold without prescription [1-2]. Their consumption is constantly growing among the general population due to widespread availability and intense advertising of OTC analgesics as safe products with proven therapeutic potential. OTC analgesics are often effective for self-management of pain. However, they are also associated with a low but important rate of gastrointestinal and hepatic events, as well as a risk of intentional and non-intentional overdose [3].

Chronic kidney disease (CKD) is a prevalent disorder, estimated in Poland at $\sim 6 \%$ of the general population [4]. Irrespective of the cause, it often leads to a state of renal insufficiency with impaired excretion of numerous metabolites and waste products. The use of OTC analgesics in this patient group could potentially be associated with an increased risk of side-effects and/or drug toxicity, due to impaired renal metabolism and/or elimination. Taking into account the above-mentioned prevalence of CKD, and the widespread use of OTC drugs in the general population, the issue of OTC analgesic use in this specific patient group seems to be of clinical importance. Therefore, the aim of this study was to evaluate the epidemiology and indications for the use of OTC analgesics in patients at various stages of CKD, in comparison to controls with preserved kidney function.

\section{Materials and methods}

A cross-sectional controlled survey study of CKD patients was conducted to evaluate their analgesic self-medication practices. The study cohort included 180 patients with CKD treated in the Department of Nephrology Transplantology and Internal Medicine of the Medical University of Gdansk. CKD was diagnosed according to KDOQI definition [5]. Inclusion criteria were restricted to the presence of CKD, age $>18$, and willingness to participate in the survey. Patients were divided into subgroups: CKD stage 1-5 (CKDND; $n=80$ ), end-stage renal failure patients treated with hemodialysis or peritoneal dialysis (CKDD; $n=50$ ), and patients after renal transplantation (CKDT; $n=50$ ). Glomerular filtration rate (GFR) was estimated based on the CKDEPI equation [6]. The control group comprised of 60 age- and gender-matched subjects without CKD, se- lected from a cohort of the Family Medicine Centre of the same hospital.

The study was based on a written anonymous survey. The questions referred to analgesic self-medication practices: frequency of analgesic use (never, sometimes, regularly i.e. $>1$ time/week), major indications, the source (advised by the doctor, by the pharmacist, by the mass media, etc.), as well as the patient's awareness of the potential side-effects of the analgesics used. Protocol of the study received approval from the Local Bioethics Committee. Results were expressed as percentages (for categorical variables), mean and standard deviation or median and interquartile range. The assumption of normality was verified with the Kolmogorov-Smirnov test. The quantitative variables' differences were assessed by t-test, analysis of variance (ANOVA) or non-parametric Kruskal-Wallis test. Differences in prevalence between selected categories were measured using the two-sample t-test test. A p-value $<0.05$ was considered to be statistically significant. Statistical processing of the results was performed with the use of the statistical software STATISTICA PL v 12.0 (Statsoft, Poland).

\section{Results}

The general demographic data of the studied groups is presented in Table 1. Patients with CKD, in general, declared greater comorbidity in comparison to subjects from the control group. Renal transplant recipients (CKDT) were, on average, younger and lived in less populated places than CKDND and CKDD patients (Table 2). Regular use of OTC analgesic drugs was reported by 35 CKD patients (18.9\%), and by 6 patients (10.0\%) from the control group; $p=0.01$. Subgroup analysis revealed that among CKD patients, CKDT reported the least frequent use of OTC analgesics (10.0\%), in comparison to CKDND (20.0\%) and CKDD subjects $(26.0 \%) ; p=0.06$. The patients from the studied groups took usually one analgesic drug at a time. However, 14 subjects $(7.8 \%)$ admitted to use at least two analgesics simultaneously. In the control group, three subjects (5.0\%) reported taking at least two analgesic drugs at the same time; $p=0.46$. The major indications for the use of OTC analgesics are presented in Table 3 . In the CKDND group, musculoskeletal issues prevailed, while headaches were the main indications in transplanted patients. Most of the patients (67.2\%) kept their doctor informed about the OTC medications used. In the control group, this percentage was slightly lower (53.3\%); $p=0.06$. Less than half of CKD patients and controls declared any knowledge on the potential side-effects of OTC analgesic drugs ( $45.6 \%$ vs. $40.0 \%$, NS). 
Table 1. Demographic data of the study groups

\begin{tabular}{|c|c|c|c|}
\hline & All CKD patients & Control group & p-value \\
\hline Number of patients ( $n$ ) & 180 & 60 & \\
\hline Male gender $(\%)$ & $101(56.1 \%)$ & $32(53.3 \%)$ & NS \\
\hline Age (years) & $58.0 \pm 15.1$ & $57.3 \pm 14.3$ & NS \\
\hline Living conditions & & & NS \\
\hline Good & $62(34.4 \%)$ & $24(40.0 \%)$ & \\
\hline Average & $108(60.0 \%)$ & $34(56.7 \%)$ & \\
\hline Poor & $10(5.6 \%)$ & $2(3.3 \%)$ & \\
\hline Education & & & NS \\
\hline Basic / Professional & $22(12.2 \%)$ & $8(13.3 \%)$ & \\
\hline Secondary & $106(58.9 \%)$ & $34(56.7 \%)$ & \\
\hline Higher & $52(28.9 \%)$ & $19(31.3 \%)$ & \\
\hline Place of residence & & & NS \\
\hline Large city $>100,000$ & $123(68.3 \%)$ & $43(71.7 \%)$ & \\
\hline Small city $<100,000$ & $36(20.0 \%)$ & $12(20.0 \%)$ & \\
\hline Village & $21(11.7 \%)$ & $5(8.3 \%)$ & \\
\hline Co-morbidities & & & NS \\
\hline $\begin{array}{l}\text { Hypertension } \\
\text { Diabetes mellitus }\end{array}$ & $123(68.3 \%)$ & $32(53.3 \%)^{*}$ & $<0.05$ \\
\hline Cardiovascular disease & $43(23.9 \%)$ & $5(8.3 \%)^{* *}$ & $<0.01$ \\
\hline Higher & $24(13.3 \%)$ & $19(15.0 \%)$ & NS \\
\hline
\end{tabular}

CKD - chronic kidney disease

NS - non-significant 
Table 2. Demographic data of the study subgroups

\begin{tabular}{|l|c|c|c|}
\hline \multicolumn{2}{|c|}{ CKDND } & CKDD & 50 \\
\hline Number of patients (n) & 80 & 50 & $50.1 \pm 12.5$ \\
\hline Age (years) & $64.2 \pm 14.7$ & $59.3 \pm 14.0$ & $<0.001$ \\
\hline Gender M: n (\%) & $41(51 \%)$ & $28(56 \%)$ & $32(64 \%)$ \\
\hline
\end{tabular}

Other diseases: $n(\%)$

\begin{tabular}{|l|c|c|c|}
\hline Diabetes mellitus & $29(36)$ & $7(14)$ & $7(14)$ \\
\hline Hypertension & $56(70)$ & $28(56)$ & $39(78)$ \\
\hline CVD & $14(17)$ & $7(14)$ & $3(6)$ \\
\hline
\end{tabular}

Education: n (\%)

\begin{tabular}{|l|c|c|c|c|}
\hline Basic & $7(9)$ & $7(14)$ & $8(16)$ & 0.62 \\
\hline Secondary & $45(56)$ & $31(62)$ & $31(62)$ & 0.88 \\
\hline Higher & $28(35)$ & $12(24)$ & $11(22)$ & 0.47 \\
\hline
\end{tabular}

Place of residence: $\mathbf{n}(\%)$

\begin{tabular}{|l|c|c|c|c|}
\hline City $>\mathbf{1 0 0 , 0 0 0}$ & $66(82)$ & $38(76)$ & $20(40)$ & 0.001 \\
\hline City <100,000 & $7(9)$ & $10(20)$ & $20(40)$ & 0.001 \\
\hline Village & $7(9)$ & $2(4)$ & $10(20)$ & 0.16 \\
\hline
\end{tabular}

Living conditions

\begin{tabular}{|l|c|c|c|c|}
\hline Good & $22(27)$ & $24(48)$ & $16(32)$ & 0.18 \\
\hline Average & $55(69)$ & $20(40)$ & $33(66)$ & 0.008 \\
\hline Poor & $3(4)$ & $6(12)$ & $1(2)$ & 0 \\
\hline
\end{tabular}

CKDND - non-dialysis dependent CKD patients (CKD stage 1-5); CKDD - dialysis patients; CKDT - patients after kidney transplantation; CVD - cardiovascular disease

Table 3. Indications for analgetics use in CKD subgroups

\begin{tabular}{|l|c|c|c|}
\hline & CKDND & CKDD & CKDT \\
\hline Musculoskeletal & $35 \%$ & $30 \%$ & $20 \%$ \\
\hline Headaches & $12 \%$ & $30 \%$ & $26 \%$ \\
\hline Abdominal pain relief & $9 \%$ & $10 \%$ & $19 \%$ \\
\hline
\end{tabular}




\section{Discussion}

The present study demonstrates that the use of OTC analgesics is higher in CKD patients, as compared to controls. This finding seems of clinical significance, given the potential toxicity of these drugs in subjects with impaired kidney function.

Intense advertising and OTC availability results in a growing consumption of analgesics worldwide. In a Canadian study, OTC non-steroidal anti-inflammatory drugs (NSAIDs) were taken by $24 \%$ of responders [7]. In Czech Republic, consumption of ibuprofen is estimated to be as high as 30 doses/1000 inhabitants/day and in Denmark consumption of paracetamol reaches 60 doses/1000 inhabitants/day [8]. The most consumed analgesic in France is paracetamol and its use increased by $140 \%$ during $2006-2015$ [9]. The doses of OTC analgesics are relatively small, and therefore the risk of side-effects is believed to be negligible. However it still exists, mainly in a form of gastrointestinal and hepatic complications $[3,10]$.

Chronic pain is a common feature of CKD [11]. Calcium-phosphate disorders leading to renal osteodystrophy, rapid changes in blood pressure during dialysis leading to headaches, and an all-together lower quality of life of CKD patients, are among the major culprits. Indeed, our study demonstrates that OTC analgesic consumption is almost twice as high in CKD patients in comparison to controls. Analysis within the CKD population revealed that there were some differences among particular subgroups of patients. While in the CKDND subjects, analgesics were taken mainly for musculoskeletal issues, in transplanted patients headache was the most prevalent indication. In CKDD patients, both these indications were similarly common. In general, analgesic use in transplanted patients was comparable to the control group, while it was twice as prevalent in CKDND and CKDD subjects. Again, the above-mentioned reasons for chronic pain in CKD could, at least in part, explain these differences in the prevalence of analgesic use and the major indications for their consumption among the studied CKD subgroups.

CKD patients differ from people with preserved kidney function. Impaired glomerular filtration rate can lead to accumulation of drugs and/or their metabolites for which the urinary tract is the primary path of elimination. Therefore, the use of OTC analgesics in this patient group might be potentially associated with a greater risk of complications, as compared to the general population. Moreover, analgesics may contribute to the progress of CKD itself. Indeed, in the XX century, analgesic nephropathy was among the major causes of end-stage renal disease (ESRD) in many countries.
In the US, as much as 7\% of ESRD cases were classified as a result of analgesic nephropathy [12], and in Australia pathological changes attributable to analgesics were observed in $5 \%$ of all the kidneys in the post mortem surveys in the general population [13]. However, based on numerous studies, it was proven that the sole medicine responsible for analgesic nephropathy was phenacetine. Either through direct nephrotoxicity or through decreasing prostaglandin synthesis, phenacetine led to chronic kidney insufficiency with erythrocyturia, increased blood pressure, and papillary necrosis found in kidney specimens [14]. Phenacetine was banned throughout the world in the second half of XX century. At present, analgesic nephropathy is not a major clinical issue any more.

Although the classic form of analgesic nephropathy is slowly fading into history, it does not mean that analgesics are completely safe for the CKD population. Manufacturers inform about the recommended and maximum doses associated with impaired kidney function in the leaflets enclosed in every drug package. For instance: metamizole dose should be decreased in case of renal insufficiency because of prolonged elimination time of the drug, and should be totally avoided in patients with GFR $<30 \mathrm{ml} / \mathrm{min}$. Severe kidney dysfunction constitutes a contraindication for the use of ibuprofen, diclofenac and paracetamol. Furthermore, NSAID use is associated with increased risk of gastrointestinal bleeding, especially in case of concurrent use of anticoagulants, as in CKDD patients who receive heparin during hemodialysis sessions. The patients are usually unaware of these limitations. In our survey, less than half declared any knowledge on the potential side-effects of OTC analgesic drugs. By using them without control, they put themselves at risk of serious and sometimes life-threatening complications.

It is not to be said that OTC analgesics should be totally avoided in CKD patients. Taking into account the chronic pain commonly experienced by CKD patients, analgesics are often the only way to improve their quality of life. However, given the scarce knowledge on potential side-effects of analgesic medications among CKD patients, it is of utmost importance to meticulously elicit the medical history of analgesic use, and keep the patients informed on the advised doses, and potential side-effects associated with their use. In our study, as much as one third of the patients did not inform their doctor about the OTC analgesic use.

The major limitations of the study are its cross-sectional character and a relatively small sample size. However, even with these groups of patients, significant differences in analgesic use were demonstrated. 


\section{Conclusion}

In conclusion, the prevalence of OTC analgesic use is higher in CKD patients than in controls, except for the transplanted subjects in whom it is similar to controls from the general population. The CKD patients' knowledge of the side-effects of OTC analgesics appears to be very limited. Taking into account the potential threats associated with the accumulation of analgesic drugs and their metabolites in the course of CKD, meticulous history-taking regarding OTC analgesics use and comprehensive patient education seems of crucial importance.

\section{References}

1. Villako P, Volmer D, Raal A: Factors influencing purchase of and counselling about prescription and OTC medicines at community pharmacies in Tallinn, Estonia. Acta Pol Pharm. 2012;69(2):335-40.

2. Sado $E$, Kassahun E, Bayisa $G$ et al.: Epidemiology of self-medication with modern medicines among health care professionals in Nekemte town, western Ethiopia. BMC Res Notes. 2017;10(1):533.

3. Bjarnason I: Gastrointestinal safety of NSAIDs and over-the-counter analgesics. Int J Clin Pract Suppl. 2013;(178):37-42.

4. Zdrojewski L, Zdrojewski T, Rutkowski M et al.: Prevalence of chronic kidney disease in a representative sample of the Polish population: results of the NATPOL 2011 survey. Nephrol Dial Transplant. 2016;31(3):433-39.

5. National Kidney F: K/DOQI clinical practice guidelines for chronic kidney disease: evaluation, classification, and stratification. Am J Kidney Dis. 2002;39(2 Suppl 1):S1-266.

6. Levey AS, Stevens LA, Schmid $\mathrm{CH}$ et al.: A new equation to estimate glomerular filtration rate. Ann Intern Med. 2009;150(9):604-612.

7. Hamilton K, Davis C, Falk J, Singer A, Bugden S: High risk use of OTC NSAIDs and ASA in family medicine: A retrospective chart review. Int J Risk Saf Med. 2015;27(4):191-99.

8. Hudec R, Bozekova L, Tisonova J: Consumption of three most widely used analgesics in six European countries. J Clin Pharm Ther. 2012;37(1):78-80.

9. Hider-Mlynarz K, Cavalie P, Maison P: Trends in analgesic consumption in France over the last 10 years and comparison of patterns across Europe. Br J Clin Pharmacol. 2018;84(6):1324-34.

10. Stiel D: Exploring the link between gastrointestinal complications and over-the-counter analgesics: current issues and considerations. Am J Ther. 2000;7(2):91-98.

11. Wu J, Ginsberg JS, Zhan M et al.: Chronic pain and analgesic use in CKD: implications for patient safety. Clin J Am Soc Nephrol. 2015;10(3):435-42.

12. Murray TG,Goldberg M: Analgesic-associated nephropathy in the U.S.A.: epidemiologic, clinical and pathogenetic features. Kidney Int. 1978;13(1):64-71.

13. Stewart JH: Analgesic abuse and renal failure in Australasia. Kidney Int. 1978;13(1):72-78.

14. Chmielewski M, Jakimowicz-Tylicka M, Rutkowski B: Analgesics - still a nephrological cause for concern? Forum Nefrol. 2015;8(2):43-48. 